\title{
Safety and Efficacy of Imatinib for Hospitalized Adults with COVID-19: A structured summary of a study protocol for a randomised controlled trial
}

\author{
Ashkan Emadi ${ }^{1,2,3^{*}}$ (D) Joel V. Chua ${ }^{2,4}$, Rohit Talwani ${ }^{2,4}$, Søren M. Bentzen ${ }^{1,5}$ and John Baddley ${ }^{1,2,4}$
}

\begin{abstract}
Objectives: Primary Objective: To evaluate the efficacy and safety of oral administration of imatinib combined with the Best Conventional Care (BCC) versus placebo plus BCC in hospitalized patients with COVID-19.

Hypothesis: Addition of imatinib to the BCC will provide a superior clinical outcome for patients with COVID-19 compared with BCC plus placebo.

This hypothesis is on the basis of 1) intralysosomal entrapment of imatinib will increase endosomal $\mathrm{pH}$ and effectively decrease SARS-CoV-2/cell fusion, 2) kinase inhibitory activity of imatinib will interfere with budding/ release or replication of SARS-CoV-2, and 3) because of the critical role of mechanical ventilation in the care of patients with ARDS, imatinib will have a significant clinical impact for patients with critical COVID-19 infection in Intensive Care Unit (ICU).

Trial design: This is an individual patient-level randomized, double-blind, placebo-controlled, two-parallel arm phase 3 study to evaluate the safety and efficacy of imatinib for the treatment of hospitalized adults with COVID-19. Participants will be followed for up to 60 days from the start of study drug administration. This trial will be conducted in accordance with the principles of the Declaration of Helsinki and the Good Clinical Practice guidelines of the International Conference on Harmonization.

(Continued on next page)
\end{abstract}

\footnotetext{
* Correspondence: aemadi@umm.edu

${ }^{1}$ University of Maryland Marlene and Stewart Greenebaum Comprehensive

Cancer Center, 22 South Greene Street, Room N9E06, Baltimore, Maryland

21201, USA

${ }^{2}$ University of Maryland School of Medicine Department of Medicine, Baltimore, MD, USA

Full list of author information is available at the end of the article
}

(c) The Author(s). 2020 Open Access This article is licensed under a Creative Commons Attribution 4.0 International License, which permits use, sharing, adaptation, distribution and reproduction in any medium or format, as long as you give appropriate credit to the original author(s) and the source, provide a link to the Creative Commons licence, and indicate if changes were made. The images or other third party material in this article are included in the article's Creative Commons licence, unless indicated otherwise in a credit line to the material. If material is not included in the article's Creative Commons licence and your intended use is not permitted by statutory regulation or exceeds the permitted use, you will need to obtain permission directly from the copyright holder. To view a copy of this licence, visit http://creativecommons.org/licenses/by/4.0/. The Creative Commons Public Domain Dedication waiver (http://creativecommons.org/publicdomain/zero/1.0/) applies to the data made available in this article, unless otherwise stated in a credit line to the data. 
(Continued from previous page)

Participants: Inclusion Criteria: Patients may be included in the study only if they meet all of the following criteria: 1) Ability to understand and willingness to sign a written informed consent document. Informed consent must be obtained prior to participation in the study. For patients who are too unwell to provide consent such as patients on invasive ventilator or extracorporeal membrane oxygenation (ECMO), their Legally Authorized Representative (LAR) can sign the informed consent, 2) Hospitalized patients $\geq 18$ years of age, 3) Positive reverse transcriptasepolymerase chain reaction (RT-PCR) assay for SARS-CoV-2 in the respiratory tract sample (oropharyngeal, nasopharyngeal or bronchoalveolar lavage (BAL)) by Center for Disease Control or local laboratory within 7 days of randomization, 4) Women of childbearing potential must agree to use at least one primary form of contraception for the duration of the study.

Exclusion Criteria: Patients meeting any of the following criteria are not eligible for the study: 1) Patients receiving any other investigational agents in a clinical trial. Off-label use of agents such as hydroxychloroquine is not an exclusion criterion, 2) Pregnant or breastfeeding women, 3) Patients with significant liver or renal dysfunction at the time of screening as defined as: 3.1) Direct bilirubin $>2.5 \mathrm{mg} / \mathrm{dL}, 3.2$ ) AST, ALT, or alkaline phosphatase $>5 x$ upper limit of normal, 3.3) eGFR $\leq 30 \mathrm{~mL} / \mathrm{min}$ or requiring renal replacement therapy, 4) Patients with significant hematologic disorder at screen as defined as: 4.1) Absolute neutrophil count (ANC) $<500 / \mu \mathrm{L}, 4.2)$ Platelet <20,000/ $\mu \mathrm{L}$, 4.3) Hemoglobin $<7 \mathrm{~g} / \mathrm{dL}, 5)$ Uncontrolled underlying illness including, but not limited to, symptomatic congestive heart failure, unstable angina pectoris, uncontrolled active seizure disorder, or psychiatric illness/social situations that per site Principal Investigator's judgment would limit compliance with study requirements, 6) Known allergy to imatinib or its component products, 7) Any other clinical conditions that in the opinion of the investigator would make the subject unsuitable for the study.

Both men and women of all races and ethnic groups are eligible for this trial.

University of Maryland Medical Center, Baltimore, MD is the initiating site. The study may be opened in other centers on the basis of the accrual rate or the magnitude of the COVID-19 pandemic.

Intervention and comparator: Imatinib: All doses of imatinib should be administered with a meal and a large glass of water. Imatinib can be dissolved in water or apple juice for patients having difficulty swallowing. In this study, patients with confirmed positive COVID-19 tests receive imatinib for a total of 14 days; 400 mg orally daily Days 1-14. Imatinib 400 mg tablets will be encapsulated using size 000 capsules and cellulose microcrystalline filler. For patients on ventilator or ECMO, imatinib will be given as oral suspension ( $40 \mathrm{mg} / \mathrm{mL})$. To make the oral suspension, imatinib tablets will be crushed and mixed in Ora-sweet solution to yield a concentration of $40 \mathrm{mg} / \mathrm{mL}$ suspension by pharmacy. Additionally, in the absence of supportive microbiological testing results, we confirm that the in-use stability period for the prepared imatinib suspensions will be 24 hours at room temperature or 7 days at refrigerated conditions. The pharmacy staff will follow the American Society Health-System Pharmacists (ASHP) guidelines for handling hazardous drugs.

Placebo: The matching placebo will be packaged by Investigational Drug Service Pharmacy at University of Maryland Medical Center. The placebos will be prepared using size 000 capsules and cellulose microcrystalline filler. Imatinib 400 mg capsules and placebo capsules will be identical form and color. For patients on ventilator or ECMO, placebo will be given as oral suspension with similar process for making imatinib suspension.

Concomitant Medications/supportive care: In both arms, patients can receive concomitant available local standard of care antipyretics, antibacterials, antivirals, antifungals and anti-inflammatory including hydroxychloroquine at the discretion of the treating physician as necessary. For other drug-drug interactions particularly with CYP P450, the treating physician should consider the risk and benefit of drug administration based on available information. Co-administration of off-label immunomodulatory treatments for COVID-19 including but not limited to corticosteroids, sarilumab, clazakizumab, tocilizumab, and anakinra will be allowed but may affect interpretability of study outcomes. The timing, dosing, and duration of these treatments will be meticulously collected, including any of these treatments that may be used for participants who experience progression of COVID-19 disease after study enrollment. Two analyses will be performed, the primary analysis will compare the primary endpoint in the two trial arms irrespective of any other treatment; the second analysis will be stratified for co-administration of immunomodulatory drugs. 
(Continued from previous page)

Main outcomes: The primary endpoint is the proportion of patients with a two-point improvement at Day 14 from baseline using the 8-category ordinal scale. The ordinal scale is an evaluation of the clinical status at the first assessment of a given study day. The scale is as follows: 1) Not hospitalized, no limitations on activities; 2) Not hospitalized, limitation on activities and/or requiring home oxygen; 3) Hospitalized, not requiring supplemental oxygen - no longer requires ongoing medical care; 4) Hospitalized, not requiring supplemental oxygen - requiring ongoing medical care (COVID-19 related or otherwise); 5) Hospitalized, requiring supplemental oxygen; 6) Hospitalized, on non-invasive ventilation or high flow oxygen devices; 7) Hospitalized, on invasive mechanical ventilation or ECMO; 8) Death.

The secondary endpoints include: All-cause mortality at Day 28, All-cause mortality at Day 60, Time to a 2-point clinical improvement difference over baseline, Duration of hospitalization, Duration of ECMO or invasive mechanical ventilation (for subjects who are on ECMO or mechanical ventilation at Day 1), Duration of ICU stay (for subjects who are in ICU at Day 1), Time to SARS-CoV-2 negative by RT-PCR, Proportion of patients with negative oropharyngeal or nasopharyngeal swab for SARS-CoV-2 by RT-PCR on days 5, 10, 14, 21, and 28 after starting treatment, Proportion of subjects with serious adverse events, Proportion of subjects who discontinue study drug due to adverse events.

The exploratory endpoints include: Determine the impact of treatment arms on IL-6 levels, Obtain blood/peripheral blood mononuclear cells (PBMCs) for storage to look at transcriptomics in severe disease, Association of major histocompatibility complex (MHC) with severity of illness, Mean change in the ordinal scale from baseline, Time to an improvement of one category from admission using an ordinal scale, Duration of hospitalization, Duration of new oxygen use, Number of oxygenation free days, Duration of new mechanical ventilation, Number of ventilator free days.

Randomization: Eligible patients will be uniformly randomized in 1:1 ratio to receive either imatinib or placebo for 14 days. Both groups will receive the BCC. The randomized treatment allocations use stratified, permuted block randomization with a variable block size; blocks are generated using a validated random number generator. In order to balance the severity of the respiratory illness between the two arms, randomization will be stratified based on radiographic findings and oxygen requirements: 1) Severe disease: evidence of pneumonia on chest X-ray or CT scan OR chest auscultation (rales, crackles), and $\mathrm{SpO}_{2} \leq 92 \%$ on ambient air or $\mathrm{PaO}_{2} / \mathrm{FiO}_{2}<300$ mmHg, and requires supplemental oxygen administration by nasal cannula, simple face mask, or other similar oxygen delivery device; 2) Critical disease: requires supplemental oxygen delivered by non-rebreather mask or high flow cannula OR use of invasive or non-invasive ventilation OR requiring treatment in an intensive care unit, use of vasopressors, extracorporeal life support, or renal replacement therapy.

Blinding (masking): The participants, caregivers, and the statistician are blinded to group assignment. The only people who are not blinded are Site Pharmacists. Blinding will be performed via a specific randomization process. Centralized, concealed randomization will be executed by the Primary Site's Pharmacist. Data on eligible consented cases will be submitted electronically on the appropriate on-study form to the pharmacy, where the patient is randomized to imatinib or placebo. Imatinib $400 \mathrm{mg}$ capsules and placebo capsules will be identical form and color. For patients on ventilator or ECMO, placebo will be given as oral suspension with similar process for making imatinib suspension.

Numbers to be randomized (sample size): The trial is designed as a double-blind, two-parallel arm, randomized controlled trial with a uniform (1:1) allocation ratio to: Arm A) Imatinib or Arm B) Placebo. Patients in both arms will receive the $B C C$ per local institutional standards at the discretion of the treating physician. Group sample sizes of 102 in Arm A and 102 in Arm B achieve 80.6\% power to detect a difference between the group proportions of 0.20 . The proportion in Arm A (imatinib treatment arm) is assumed to be 0.30 under the null hypothesis and 0.50 under the alternative hypothesis. The proportion in Arm B (placebo control arm) is 0.30 . The test statistic used is the two-sided Fisher's Exact Test. The significance level of the test is targeted at 0.05 . The significance level actually achieved by this design is $a=0.0385$. The power of the test is calculated using binomial enumeration of all possible outcomes.

The primary analysis will be conducted using an intention to treat principle (ITT) for participants who at least receive one dose of study drug or placebo. The sample size is not inflated for dropouts. All patients will be (Continued on next page) 
(Continued from previous page)

evaluable irrespective of the clinical course of their disease.

Trial Status: Current protocol version is 1.2 from May 8, 2020. The recruitment started on June 15, 2020 and is ongoing. We originally anticipated that the trial would finish recruitment by mid 2021. We are aware of the enrollment requirement of approximately 200 patients, which is required to provide scientific integrity of the results. We are also aware of the fact that enrolling this number of patients in a single-site at University of Maryland Medical Center (UMMC) may take longer than expected, particularly taken into account other competing studies. For this reason, we are actively considering opening the protocol in other sites. After identification of other sites, we will fulfill all regulatory requirements before opening the protocol in other sites.

Trial registration: ClinicalTrials.gov Identifier: NCT04394416. First Posted: May 19, 2020; Last Update Posted: June 4, 2020. FDA has issued the "Study May Proceed" Letter for this clinical trial under the Investigational New Drug (IND) number 149239.

Full protocol: The full protocol is attached as an additional file, accessible from the Trials website (Additional file 1). In the interest in expediting dissemination of this material, the familiar formatting has been eliminated; this Letter serves as a summary of the key elements of the full protocol.

Keywords: COVID-19, Randomised controlled trial, protocol, SARS-CoV, imatinib, tyrosine kinase inhibitor, TKI, lysosomal sequestration, viral ABL-1 signalling, TKI-induced attenuation of vascular leak, TKI-induced prevention and treatment lung inflammation

\section{Supplementary information}

Supplementary information accompanies this paper at https://doi.org/10. 1186/s13063-020-04819-9.

\section{Additional file 1.}

\section{Acknowledgements}

Not applicable.

\section{Authors' contributions}

AE conceived the study, created the design, secured funding, obtained regulatory requirements, and drafted and wrote the typescript. JVC, RT, JB read and were involved in critical appraisal and revision of the Protocol. SMB designed the statistical analysis methods, calculated the sample size and created the randomization table. All authors approved the final protocol.

\section{Funding}

The study is funded by the University of Maryland Greenebaum Comprehensive Cancer Center. The funding body had no role in the design of the study and collection, analysis, and interpretation of data and in writing the manuscript.

\section{Availability of data and materials}

The main principal investigator and the corresponding author (AE) and other principal investigators (JVC, RT, JB) will have access to the final trial dataset. The corresponding author will evaluate any request for data sharing and will consult with the other principal investigators after the publication of the main results. Requests can be sent to aemadi@umm.edu.

\section{Ethics approval and consent to participate}

University of Maryland, Baltimore (UMB) Institutional Review Board (IRB) approved the the referenced protocol entitled, "2038GCCC: Randomized Double-Blind Placebo-Controlled Trial on the Safety and Efficacy of Imatinib for Hospitalized Adults with COVID-19". Approval for this project is valid from $4 / 30 / 2020$ to $4 / 29 / 2021$

The principal investigator and the corresponding author (AE) certifies that this trial has received ethical approval from the appropriate ethical committee as described above.

Informed consent is a process that is initiated prior to the individual agreeing to participate in the study and continues throughout the individual's study participation. The informed consent form is IRB-approved and the subject will be asked to read and review the document.
Due to the excessive risk of COVID-19 transmission and to preserve limited personal protective equipment (PPE) for essential procedures, a remote consenting process can be used. The following considerations for remote informed consenting should be followed: The investigator and/ or his designee is responsible for presenting the risks and benefits of study participation to the subject in simple terms using the informed consent document; Potential subjects will be given an informed consent form (either paper or electronic document in a portable electronic device) and allowed time to read this document; The elements of the informed consent, including discussion of the risk and benefits, will be done either over the phone or via remote video conferencing methods approved by the IRB; Research team member will document how the consent form was transmitted or given to participant, and what method was used to communicate the informed consent; The investigator will ensure that written informed consent is obtained from each subject or legally authorized representative by obtaining the appropriate signatures and dates on the informed consent document before the performance of protocol evaluations or procedures; Signatures may be obtained using the following options: (1) electronic signature, or (2) photograph of signature/signature page sent back to study team; Subjects must date the informed consent form to be valid; Study team member should document how signature was obtained.

If unable to obtain a participant signature on an informed consent document, the following should be followed: Document the method used for communication with the patient; Document means by which agreement was communicated; Document that no imaging technology was available to capture a signed consent form; A witness to the process AND a witness to sign and date the informed consent form.

Participants must be informed that participation is voluntary and that they may withdraw from the study at any time, without prejudice. Copies of the informed consent documents will be given to the participants for their records. The informed consent process will be conducted and documented in the source document (including the date) before the participant undergoes any study-specific procedures. The rights and welfare of the participants will be protected by emphasizing to them that the quality of their medical care will not be adversely affected if they decline to participate in this study. For patients who are too unwell to provide consent such as patients on invasive ventilator or ECMO, Legally Authorized Representative (LAR) can sign the informed consent.

Consent for publication

Not applicable. 


\section{Competing interests}

The authors declare that they have no competing interests.

\section{Author details}

'University of Maryland Marlene and Stewart Greenebaum Comprehensive Cancer Center, 22 South Greene Street, Room N9E06, Baltimore, Maryland 21201, USA. ${ }^{2}$ University of Maryland School of Medicine Department of Medicine, Baltimore, MD, USA. ${ }^{3}$ University of Maryland School of Medicine Department of Pharmacology, Baltimore, MD, USA. ${ }^{4}$ University of Maryland School of Medicine, Institute of Human Virology, Baltimore, USA. ${ }^{5}$ University of Maryland School of Medicine Department of Epidemiology and Public Health, Baltimore, MD, USA.

Received: 14 October 2020 Accepted: 16 October 2020

Published online: 28 October 2020

\section{Publisher's Note}

Springer Nature remains neutral with regard to jurisdictional claims in published maps and institutional affiliations.

Ready to submit your research? Choose BMC and benefit from:

- fast, convenient online submission

- thorough peer review by experienced researchers in your field

- rapid publication on acceptance

- support for research data, including large and complex data types

- gold Open Access which fosters wider collaboration and increased citations

- maximum visibility for your research: over $100 \mathrm{M}$ website views per year

At $\mathrm{BMC}$, research is always in progress.

Learn more biomedcentral.com/submissions 